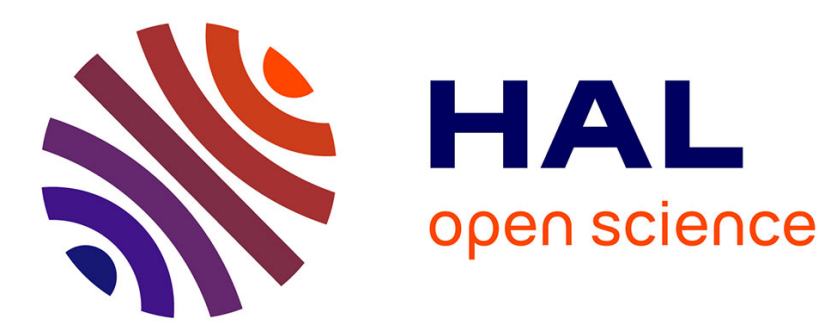

\title{
Migrants dans une banlieue beyrouthine
}

\author{
Marie-Antoinette Hily
}

\section{To cite this version:}

Marie-Antoinette Hily. Migrants dans une banlieue beyrouthine. Maghreb-Machrek, 2009, 199, pp.6170. 10.3917/machr.199.0061 . halshs-00644332

\section{HAL Id: halshs-00644332 \\ https://shs.hal.science/halshs-00644332}

Submitted on 24 Nov 2011

HAL is a multi-disciplinary open access archive for the deposit and dissemination of scientific research documents, whether they are published or not. The documents may come from teaching and research institutions in France or abroad, or from public or private research centers.
L'archive ouverte pluridisciplinaire HAL, est destinée au dépôt et à la diffusion de documents scientifiques de niveau recherche, publiés ou non, émanant des établissements d'enseignement et de recherche français ou étrangers, des laboratoires publics ou privés. 


\title{
MIGRANTS DANS UNE BANLIEUE BEYROUTHINE
}

\author{
Marie-Antoinette HIL Y*
}

La lecture des travaux consacrés aux migrations au Moyen-Orient permet d'esquisser le bilan du développement massif des migrations de populations non arabes. Il montre que ces populations suscitent et introduisent des changements dans les espaces urbains. Les avancées dans la description des mobilités spatiales et sociales qui en découlent restent cependant insuffisantes pour comprendre la manière dont s'opèrent ces transformations et le rôle et la place qu'y occupent les migrants. Il existe en effet peu de travaux sur les modes d'insertion des populations migrantes dans la ville et sur les relations qu'elles entretiennent avec ses différents « lieux » ou " milieux », malgré le caractère plus ou moins durable de nombre de leurs implantations. Comment ces nouveaux travailleurs et surtout travailleuses dont les statuts du point de vue juridique sont divers ( « réfugiés », " travailleurs temporaires », " sanspapiers » etc.) sont-ils devenus visibles dans la ville et plus précisément dans certains quartiers, et en quoi leur présence met-elle à l'épreuve des socialisations pré-établies ou historiquement constituées?

Cet article se propose de rendre compte, à partir d'enquêtes de terrain, des signes, marques etc. qu'inscrivent les nouveaux migrants dans le paysage urbain et dans l'espace public d'une banlieue de Beyrouth, Bourj-Hammoud. L'entrée par l'observation et l'entretien permettront de rendre compte des pratiques des " nouveaux venus », de leurs usages de la ville et de leurs actions dans la ville. Il s'agira aussi d'apprécier les espaces d'interaction ou de coprésence, les relations de travail, les conduites d'évitement ou d'attention entre les «locaux » et les « migrants ». C'est donc l'arrivée à la visibilité qu’il s'agit d'interroger comme " événement » dans les routines de citadins d'une part, et comme indice d'une mondialisation « en dessous » selon l'expression de Samuel Bordreuil.

\section{LE LIBAN PAYS D’IMMIGRATION?}

Depuis 1990, date de la fin du conflit au Liban, on assiste à une augmentation des besoins en main-d'oeuvre suite aux conséquences de la guerre (émigration des Libanais ${ }^{1}$, reconstructions, dégradation des salaires, turbulences politiques, etc.) et plus récemment suite

\footnotetext{
${ }^{*}$ Chercheure CNRS, MIGRINTER - Université de Poitiers / CNRS : UMR 6588

${ }^{1}$ Estimée en 1991 à 2,5 millions, la diaspora libanaise aurait été en 2005 de l’ordre de 13 millions (y compris les descendants), dont 10,7 millions en Amérique du Sud et en Amérique du Nord, 1,2 million en Afrique, 400000 en Europe, 400000 dans les pays arabes et 300000 en Australie. Voir Gérard-François Dumont, " Les populations au Liban » dans Outre-Terre, n 13 2005/4 pp. 419-445.
} 
au « retrait syrien ». Le Liban est donc devenu ces dernières années un espace d'accueil pour des milliers des travailleurs en provenance du sous-continent indien et du Sud-Est asiatique auquel s'ajoutent des personnes " réfugiées " venant d’Irak, du Soudan, de Somalie notamment et qui arrivent via la Syrie (ce pays ne demandant pas de visas d'entrée aux ressortissants de pays arabes). On ne saurait, en l'absence de chiffres fiables, évaluer le nombre d'étrangers travaillant au Liban même si certaines sources avancent le chiffre de 500 000 dont 80000 en situation régulière ${ }^{2}$. On constate aussi que la migration féminine est en augmentation. Ainsi, selon Ray Jureidini ${ }^{3}$, on estime à 100000 le nombre de Sri Lankaises, à 30000 le nombre de Philippines et à 15000 le nombre d'Éthiopiennes. En 1999, toujours selon cet auteur, $95 \%$ des ressortissants philippins au Liban étaient de sexe féminin et travaillaient comme domestiques (la population active est estimée à 1500000 personnes au Liban). Ces immigrés récents subissent de très mauvaises conditions de travail et vivent dans une précarité permanente, occupant des emplois dans les secteurs des services et de la construction. On recense globalement deux catégories de main-d'oeuvre : les employés sous contrat de travail temporaire, recrutés par des agences privées et dépendant d'un seul employeur (le garant), et une main-d'œuvre dite " illégale » (permis expirés ou non renouvelés, entrées clandestines, visas de tourisme expirés, départ clandestin de chez l'employeur).

En l'absence de droits dont les institutions étatiques seraient le garant, nombre de rapports émanant d'ONG libanaises ont entrepris de dénoncer les conditions de travail dont sont victimes ces travailleurs ${ }^{4}$. Les seules instances de recours face aux manques de l'État sont en effet les ONG, dont le Comité pastoral des Migrants afro-asiatiques dirigé par Caritas, et dans une moindre mesure les ambassades. Ce sont d'ailleurs ces ONG qui ont contribué à rendre visible cette population en se manifestant sur la scène publique lors du raz-de-marée de 2004 qui ravagea les côtes de l’Indonésie, du Sri Lanka, des Philippines et de l'Inde, pays d'origine des migrants. Cet événement a mobilisé la communauté des Franciscains et des réseaux catholiques qui ont largement contribué au retour de nombreuses femmes sans passeport et sans moyens. Les enquêtes réalisées par le centre des Migrants de Caritas Liban ont depuis alerté sur les mauvais traitements ${ }^{5}$ infligés aux travailleurs par les employeurs dénonçant ainsi la faiblesse de l'État de droit et appelant avec le soutien de quelques intellectuels ${ }^{6}$, à la mise en place d'une politique d'immigration. Depuis, de nombreuses actions ont été menées en faveur des migrants ${ }^{7}$ mais l'événement le plus marquant, celui qui a de nouveau attiré l'attention des médias ( $L^{\prime}$ Orient - Le Jour ${ }^{8}$ ) et des associations sur la

\footnotetext{
${ }^{2}$ Voir « Quelle protection pour les travailleurs migrants au Liban ? », www.franciscansinternational.org/letters/Liban.doc

3 Ray Jureidini, «L'échec de la protection de l’État : les domestiques étrangers au Liban » dans Revue Européenne des Migrations Internationales, 2003, 19-3, pp. 95-128. http://remi.revues.org/485

${ }^{4}$ R. Jureidini, op.cit. ; Agnès Deboulet, Éthiopiennes, Philippines et Soudanais : voisinages migrants et confrontation aux sociétés d'accueil à Beyrouth, Communication à l'Association Française de Sociologie, RT 2, Bordeaux, 5 septembre 2006 ; Julien Bret, Circulations transnationales et travail disqualifié au Moyen-Orient. Les travailleurs non arabes au Liban, Hommes et Migrations, 2007, n 1266, pp. 96-107 ; Knut Bergem, The Role of the State in the in-Migration of Domestic Workers to Jordan and the GCC Countries, in De Bel-Air Françoise (dir.), Migration et politique au Moyen-Orient, IFPO, Beyrouth, 2006, pp. 61-79.

${ }^{5}$ En 2006, le film de Carol Mansour, Maid in Lebanon financé par Caritas Suède a contribué à rendre publique la situation des domestiques étrangères à Beyrouth. Il y dénonçait les pratiques des employeurs et des agences, qui se sont multipliées à Beyrouth ces dernières années, à l'égard des domestiques.

${ }^{6}$ Membres de Frontiers Center, organisation qui intervient pour défendre les réfugiés non palestiniens emprisonnés.

${ }^{7}$ Rencontre organisée par COATNET (réseau d'organisations chrétiennes contre la traite des humains).

${ }^{8}$ Presse francophone
} 
situation des " migrantes », a eu lieu en 2006 et a mis en scène sur fond de guerre plus de 13000 personnes qui souhaitaient fuir le Liban bombardé ${ }^{2}$. Mais toutes ne sont pas parties et n’ont pas profité de cette opportunité choisissant de rester, préférant l'incertitude de leur sort à l'incertitude d'un retour et à l'aventure d'un départ vers un pays que nombre d'entre elles ont quitté depuis de nombreuses années. Ce fut notamment le cas de femmes, philippines pour la plupart, qui ont réussi à se « construire une vie » à Beyrouth ${ }^{10}$.

C'est dans un contexte libanais extrêmement instable donc que ces populations, pauvres et sans droits, arrivent à visibilité sociale. Qu'ils soient isolés ou regroupés, ces migrants non arabes dits " temporaires » ou " en transit », mais dont certains sont là depuis une dizaine d'années, ont fait leur entrée sur le marché du travail et tentent de s’inscrire, pour nombre d'entre eux, dans un « milieu » aux identités multiples et conflictuelles.

\section{BOURJ-HAMMOUD : DES MIGRANTS VISIBLES}

Présentée comme « le grand marché de Beyrouth », Bourj-Hammoud, se situe à 2 km du centre de Beyrouth sur la côte méditerranéenne. Cette municipalité ${ }^{11}$ compte environ 150 000 habitants à majorité chrétienne arménienne ${ }^{12}$. Si, au début du XXe siècle, BourjHammoud $^{13}$ n'était qu'un village agricole, l'arrivée des Arméniens va progressivement contribuer à son développement. C'est sous le mandat français que trois vagues de réfugiés arméniens (1918, 1922 et 1939) venant respectivement de l’Arménie, de la Turquie et du Sandjak d'Alexandrette bâtirent initialement ce qu'ils désignaient comme « la petite Arménie " et que nombre de Libanais arméniens du quartier appellent encore « le camp St Jacques ». Aux ruelles étroites bordées de petites maisons en dur, blanchies à la chaux, ce qui reste de cet îlot aujourd'hui abrite encore de vieilles familles arméniennes aux faibles ressources. Le père Mécérian donne un aperçu des conditions de vie des premiers venus : " Avec des planches de fortune, on fabriqua des baraques... une cité ouvrière se forma, spontanément, avec toutes sortes de métiers. Les femmes qui le pouvaient allaient travailler en ville. Les jeunes filles s'usaient les prunelles à broder, à faire des dentelles contre une rétribution journalière très modique $^{14} »$, alors que les hommes s'employaient comme maçons ou manouvriers. Mais la communauté va peu à peu se diriger vers le commerce d'articles de confection cousus par les femmes et les enfants puis développer un artisanat de chaussures et surtout de bijoux et étendre son activité à la maroquinerie et à l’horlogerie. Après 1948 et 1967 (guerres israélo-

\footnotetext{
${ }^{9}$ Originaires du Sri Lanka (5 370), des Philippines (4 046), d'Éthiopie (2 410), du Bangladesh (727), du Vietnam (77), du Népal (26), du Soudan (436), du Ghana (62), du Cameroun (11), de Madagascar (4), du Maroc (7) et enfin des Seychelles (6), des femmes employées comme domestiques, sans passeports (soustraits par les employeurs) et souvent sans ressources (salaires non payés) ont été rapatriées avec l'aide de l'OIM (données de l'OIM).

${ }^{10}$ Après la fin du conflit plus de 500000 migrants non arabes sont entrés sur le territoire libanais (Chiffres recueillis par Suzanne Menhem auprès de la Sûreté générale en 2007).

${ }^{11}$ Voir Khayat Tristan, « Construction du territoire municipal et aménagement métropolitain à Borj Hammoud » dans Agnès Favier, Municipalités et pouvoirs locaux au Liban, Beyrouth, CERMOC, coll. « Les Cahiers du CERMOC », 2001, n²5, p. 207-225.

${ }^{12}$ On comptabilise entre 150000 et 200000 Arméniens au Liban sur une population d'environ 3,5 millions de personnes.

${ }^{13}$ Dans les années 1960 et 1970 Bourj-Hammoud a acquis la réputation d'être un lieu de « délinquance et de débauche ». La nuit tombée, écrit L. Buccianti (Le commerce à Bourj-Hammoud, mémoire de maîtrise, université Saint-Joseph, Beyrouth, 1972), « les baraques à sandwichs se transforment en tripots de jeux dirigés par les abadayes de la région ».

${ }^{14}$ Liliane Buccianti, op. cit.
} 
arabes), Bourj-Hammoud va accueillir de nouvelles populations, des Libanais du sud mais aussi des réfugiés kurdes et syriens ${ }^{15}$.

La grande transformation de Bourj-Hammoud en quartier commerçant va se faire entre 1950 et la fin des années 1960. Le quartier va s’organiser progressivement autour de rues commerçantes spécialisées dans différents secteurs : alimentation, chaussures, cuir, stations d'essence, matériel mécanique, sous-agences d'automobile japonaises et françaises, banques (Transorient Bank, Federal Bank, Crédit Populaire ${ }^{16}$ ). Ce n’est qu'après les années de guerre, que Bourj-Hammoud, qui accueillera des commerçants arméniens chassés du centre de Beyrouth (les déplacés), verra son activité de commerce se redéployer.

Mais depuis une dizaine d'années ce sont de nouveaux migrants qui se sont inscrits dans le paysage de Bourj-Hammoud (entre un et trois mille selon différentes estimations. Il est, évidemment, difficile d'en faire un comptage précis, d'autant que les mobilités sont importantes). Sri Lankais, Philippins, Bangladais, Indiens Tamouls, Éthiopiens, Égyptiens, Ghanéens, Syriens, Soudanais, Kurdes, Béninois et Sénégalais sont désormais visibles dans ce quartier et plus particulièrement à Dora, nouvelle centralité beyrouthine ${ }^{17}$, animée et bruyante. Nombre de ces migrants sont en situation illégale (3\% selon la police ${ }^{18}$; chiffre fortement sous-évalué selon d'autres interlocuteurs) et occupent des emplois précaires : domestiques, employés dans les métiers du bâtiment, employés dans des magasins (coursiers) ou dans des restaurants (serveurs, cuisiniers, plongeurs, balayeurs), employés dans l'industrie (charcuterie arménienne, fabrication de la bière Almaza, fabrique de fours à pain exportés dans les pays arabes) ouvriers dans des fabriques de chaussures, de vêtements de cuir, de confection, de sacs plastiques et enfin employés dans des garages. Ces personnes, dont une bonne partie ne travaille pas dans la commune, mais à Beyrouth centre et ouest, ont trouvé dans cet espace urbain aux immeubles à plusieurs étages et à l'habitat serré et dense, qui ne dénotent apparemment ni l'aisance ni la misère, des occasions, de se loger dans des appartements délabrés et mal entretenus ${ }^{19}$, ce qu’elles paient chèrement.

Que retirer de cette brève description de l'histoire du quartier, rapportée par quelques commerçants, quelques notables, un religieux, de jeunes employés, etc. au hasard des rencontres et des demandes. Chez les plus anciens habitants c'est le rôle des Arméniens ${ }^{20}$ dans la construction du quartier qui est mis en avant. Ils soulignent tous un avant et un après de la consistance de la communauté arménienne, un avant et un après la guerre mais aussi un avant et un après de la prospérité du quartier. Ce qui a beaucoup changé nous dit Monsieur A : «C'est la guerre de 75 : tout a été bousculé et plus rien n'est comme avant. Vous savez qu'ici pendant une époque il y avait un casino, des restaurants près de la mer... on était prospère et le travail comptait. Maintenant, c’est encore autre chose... les étrangers on les emploie pour

\footnotetext{
${ }^{15}$ L'agglomération beyrouthine décrite par Samir Kassir (Histoire de Beyrouth, Fayard, 2003, 732 p.) voisinait avec la « ceinture de misère » : bidonvilles, dans lesquels on trouvait des Syriens et des Kurdes de Turquie mais aussi des Palestiniens. Voir Revue Géographique de Lyon : "Les bidonvilles de Beyrouth », A. Bourgey, J. Phares, Lyon, 1973.

${ }^{16}$ Ces commerces voisinent avec une vente de sandwiches et de boissons dans des baraques améliorées datant des premières arrivées d'Arméniens.

${ }^{17}$ Pendant la guerre, les services bancaires se sont « décentralisés » à Dora, quartier chrétien, alors que le centre de Beyrouth était paralysé par les affrontements. Depuis une dizaine d'années ce quartier est devenu un nouveau centre de commerce et d'affaires.

${ }^{18}$ Entretien avec un responsable des services de la police de Bourj-Hammoud.

${ }^{19} \mathrm{Si}$ la présence des travailleurs étrangers est importante à Bourj-Hammoud, d'autres quartiers populaires comme ceux du sud de Beyrouth accueillent aussi ces nouveaux migrants.

${ }^{20}$ Les Arméniens sont citoyens libanais.
} 
pas cher, ce sont des travailleurs, maintenant il n’y a pas encore de problèmes avec eux mais bon... ». Si ces vieux artisans, qui ont fait la réputation de Bourj-Hammoud comme haut lieu du commerce, ont la nostalgie d'une époque où " le métier était un capital », en réponse à l'interrogation sur la présence des migrants, ils évoquent les épreuves qu'ont connues les Arméniens pour construire leur quartier. Quand on parle des étrangers, c'est bien les premiers installés qui parlent d'eux.

\section{MIGRANTS EN MOUVEMENT : LA RUE D’ARMÉNIE}

L’échelle de la rue comme espace privilégié de la multiplicité des temps et des acteurs devient un observatoire pertinent des mouvements de l'étranger et vitrine de la réputation du quartier. Le rapport entre le quartier et sa rue centrale permet d'illustrer quels changements introduit l'étranger, et comment un sentiment d'appartenance à un quartier est mis à l'épreuve par l'introduction de la différence.

Au début de la rue d'Arménie, la Western Union avec sa devanture jaune et noire où l'on peut lire the fastest way to send and receive money worldwide répond aux propos d'un vieux tailleur arménien " ce quartier c'est comme en Amérique, il y a beaucoup d'étrangers ». Et juste à côté de cette échoppe poussiéreuse, des commerces sri lankais, proposent des cassettes de musique indienne, des textiles indiens, des produits d'épicerie, alors qu'à l'étage supérieur, le " Pamma mini market » offre une restauration aux habitués qui se retrouvent le dimanche devant un écran de télévision diffusant en boucle « les meilleurs clips bollywood en hindî et en ourdou ».

Dans la rue d'Arménie, la circulation humaine et la densité des badauds signalent la concentration des échanges économiques et commerciaux. Les marques qu'impriment les Sri Lankais, Indiens, Philippins à l'espace de la rue sont visibles, témoignages des changements que connaît Bourj-Hammoud, quartier où les commerçants arméniens restent cependant dominants. La rue d'Arménie est diversement fréquentée et utilisée : espace ouvert où l'on s'active et se montre soigneusement vêtu le dimanche, jour de repos et d'approvisionnement, espace des hommes du quartier, installés aux terrasses des bistrots-trottoirs, espace des migrants qui s’approvisionnent dans les magasins philippins ou sri lankais, et dans les étals de rue où l'on peut acheter les dernières productions musicales indiennes. Mais aussi, espace des transactions monétaires que les migrants opèrent dans les boutiques de transferts de fonds, tenues par des Libanais dont les employés traitent dans les différentes langues des clients (anglais, hindi, singhalais). Les " nouveaux » commerçants se sont insérés dans ce tissu commercial à la faveur de contrats informels passés avec des propriétaires libanais (le montant de la location est de l'ordre de 7000 dollars par an déclarés par les propriétaires) et s'approvisionnent indifféremment dans les pays d'origine ou chez un grossiste spécialisé dans l'import de marchandises en provenance d'Inde et du Sri Lanka. Aujourd'hui, une dizaine de ces magasins-restaurants peuvent offrir des produits qui répondent aux demandes des différents groupes. La rue d'Arménie attire aussi une clientèle de migrants employés dans Beyrouth-centre, qui, le dimanche, viennent remplir leur journée, occupés à consommer, téléphoner à la famille des cabines nichées devant les bureaux de change. À l'heure du repas les restaurants indiens, sri lankais et philippins reçoivent des Philippines qui ont assisté à un office dans une salle transformée en église à l'étage d'un centre associatif géré par des sœurs libanaises, ou des Indiens qui se sont retrouvés dans un espace de réunion pour pratiquer leur religion, le bouddhisme. 
Si aujourd'hui « on trouve de tout » dans la rue d'Arménie, on trouve essentiellement des produits importés de Taïwan et de Chine dans de vastes magasins (les enseignes, $1 \$$ SHOP ou 500 L.L. Big solde, avertissent le passant), dans des boutiques d'appareils photos numériques, de portables, de matériel $\mathrm{Hi}-\mathrm{Fi}$, etc. Bien plus que les marques très visibles qu'impriment les Sri Lankais, Indiens, Philippins à l'espace de la rue, les commerçants arméniens s'insurgent contre ces grands magasins à bas prix qui, selon les propos de l'un d'eux, vont chasser les petits commerces traditionnels, indice aussi du passage d'une forme de sociabilité de quartier, assimilé à une communauté, où les statuts étaient stables à la mobilité des statuts et à un ébrèchement de cette même communauté. Quant aux commerces tenus par des étrangers, le discours est variable : « c'est à la fois bien et pas bien » dit-on. Et par « bien » ce commerçant entend « leur capacité à développer le commerce et à respecter la loi » et le « pas bien » est adressé à ceux qui transgressent la loi, et particulièrement à l'encontre de ceux qui pourraient donner du quartier une mauvaise image.

Que les sociabilités dans la rue d'Arménie ne se résument qu’à une coprésence de tous ceux qui la fréquentent ne fait guère de doute, même si quelques rares couples mixtes marchent côte à côte. Reste que, si les habitants du quartier disent que ces populations ne « gênent » pas, ils constatent qu'après le départ récent des Syriens, une autre " clientèle » est en mesure d'occuper les appartements que les classes moyennes désertent. La reprise des commerces délaissés par les Libanais arméniens est souvent bienvenue et rentable. Et même si les migrants sont encore perçus comme de passage, Guest Workers, " en transit ", il n'est pas rare de rencontrer des Philippines qui travaillent à Beyrouth depuis de nombreuses années et qui ont aménagé leur vie ici tout en maintenant le lien avec la famille là-bas qu'elles contribuent largement à faire vivre.

Les Philippines que l'on rencontre le dimanche, rue d'Arménie dans une agence de transfert d'argent ou chez un photographe spécialisé dans les portraits ou encore chez un bijoutier, négociant en arabe des petits bijoux en or, se retrouvent le plus souvent dans de grands magasins. Pour quelques dollars, elles achètent des cadeaux destinés à la famille et surtout aux enfants restés au pays. Quelques-unes d'entre elles fréquentent un restaurantépicerie tenu par un Libanais à la retraite qui emploie celle que l'on appelle " la patronne ». Cette Philippine, au Liban depuis dix ans, fait la cuisine, conseille sur les produits à base de plantes, fabriqués en Chine, qu'achètent ses compatriotes en grande quantité pour les revendre au pays quand elles y retournent. Elle vend aussi des bijoux qu'elle présente à la demande mais surtout elle endosse le rôle de confidente auprès des démunies. Avant de s'installer à Bourj-Hammoud, elle avait travaillé à Hong Kong, puis après avoir été domestique pendant deux ans dans une famille libanaise, elle a réussi à " s'émanciper » de son kafil (garant) pour travailler en free lance. Elle envoie régulièrement de l'argent à ses cinq enfants dont deux sont mariés. Les jeunes femmes qui se réunissent dans cet établissement font des ménages à l'heure après avoir travaillé dans des hôtels ou chez des particuliers. Certaines qui ont réussi à fuir leurs employeurs (runaway(s)) vivent en général à plusieurs dans le sud du quartier, là où les appartements sont le moins cher. Toutes ces femmes circulent et font en quelque sorte leur propre travail de terrain pour trouver du travail, entrer en relation, chercher aide et soutien, s'allier avec des compatriotes pour constituer des espaces de sociabilités. Si leur présence ne va pas de soi (« les immigrés ${ }^{21}$ sont tolérés mais pas acceptés, ils ont mis des problèmes dans la société libanaise » selon les propos d'un commerçant), leur présence paraît cependant de moins en moins déplacée.

\footnotetext{
${ }^{21}$ On remarquera que ce Libanais qui s'exprime en français utilise le terme « d'immigré ».
} 
De façon générale à Bourj-Hammoud les migrants ne sont pas protégés d’un racisme qui n'est qu'à peine masqué. Ainsi, un de mes interlocuteurs établira une hiérarchie où selon ses propres termes " en bas de l'échelle on trouve les Noires, et en haut de l'échelle les Philippines ", réputées propres et bonnes épouses : " Ces filles viennent en général d'un milieu rural et celles qui s'enfuient le font soit parce que le travail demandé est trop lourd, soit pour des raisons personnelles. Elles pensent que c'est plus facile et qu'elles peuvent travailler pour plus d'argent et sans papiers, quand bien même elles sont sur les " black lists » des agences. Les Philippines sont très appréciées car elles élèvent bien les enfants et sont des bonnes épouses... les Noires n’ont pas le profil qui plaît aux Libanais et elles ne sont pas appréciées. Les Philippines ont donc plus de possibilités pour trouver des hommes » (Monsieur B, 50 ans, Libanais arménien). Et si on signale environ $10 \%$ d'unions mixtes dans le quartier, c'est majoritairement entre Arméniens libanais et Philippines.

Les nouveaux « résidents » étrangers vivent dans la précarité, et quand ils se déplacent c'est dans la peur du contrôle ${ }^{22}$. Pourtant dans cette incertitude quotidienne, s'organise une vie sociale avec l'espoir de "s'en sortir » ou plus justement de trouver des moyens de gagner de l'argent, contraints que sont les migrants d'accepter des conditions de travail difficiles et des emplois mal rémunérés. Ici les " migrants » et particulièrement les " migrantes » s'activent à conjuguer nécessité de la discrétion et de l'évitement et rencontre avec les « locaux/nationaux », ceux qui se revendiquent comme Libanais, Arméniens ou Libanoarméniens.

Alors que le quartier, caractérisé par des mobilités lentes et maîtrisables et des solidarités construites dans l'épreuve de l'exil, est évoqué avec nostalgie par les plus anciens des habitants arméniens, les mêmes perçoivent les « migrants » comme venant bousculer un monde qui leur échappe et se transforme et dans lequel ces « migrants » viennent jouer leurs propres jeux.

Le constat que font ces vieux résidents renvoie à des observations bien connues pour nos villes occidentales et que souligne Alain Tarrius ${ }^{23}$ quand il fait référence à la construction de " région de moeurs » (" cette conjonction entre désir individuel et contrainte objective qui se négocie dans la ville, qui délimite des espaces éphémères, à partir de mobilités surprenantes, inhabituelles pour des populations d'origine, de statuts et de situations différentes et qui se trouvent dans le même lieu pour partager un peu ces désirs-là »).

\section{POUR CONCLURE}

La rue d'Arménie a quelque chose de familier, de déjà-vu. Les situations de coprésence observées, mais aussi les signes d'alliances commerciales, et parfois privées, renvoient à d'autres situations elles-mêmes observées dans d'autres villes, et il est permis de s’interroger sur le caractère singulier ou « spécifique » de ce qui se passe à Bourj-Hammoud. Ces «mobilités » transnationales s'inscrivent, comme dans d'autres régions du monde, dans ce qu'il est convenu d'appeler « la mondialisation de la force de travail » ou la « mondialisation migratoire » Et ce que l'on appelle aussi les lieux « cosmopolites » ici comme

\footnotetext{
${ }^{22}$ Les étrangers n'ayant pas de papiers en règle sont passibles d'une peine d'emprisonnement et d'une amende. Voir O. Clochard et M. K Doraï, « Aux frontières de l'asile, les réfugiés non palestiniens au Liban », A contrario, vol. 3, n 2, 2005

${ }^{23}$ Alain Tarrius, La mondialisation par le bas, les nouveaux nomades de l'économie souterraine, Paris, Balland, 2002, $168 \mathrm{p}$
} 
ailleurs donnent à voir ce que l'on sait depuis Simmel, à savoir que la ville est une " formidable machine à multiplier les différences, que ce soit dans les produits (ceux amenés par les commerçants étrangers) ou les types de services qu'elle encourage aussi bien que dans les « rameaux culturels » qu'elle déplie dans l'entresoi des cercles d'affinités ${ }^{24}$ ». Mais dans ces espaces, il est permis de s'interroger sur nos catégories d'intégration, d'inclusion/exclusion, de « population en transit », etc. Font-elles sens ?

Les migrants de Bourj-Hammoud investissent les lieux, sans se les approprier pourtant, et mobilisent des microréseaux pour créer de la richesse. Qu'ils ne soient que de passage (en " transit », notion elle aussi plus qu'incertaine) ou qu'ils se stabilisent pour un temps ou pour plus longtemps, les migrants par leur présence, leur visibilité et leurs pratiques, donnent du contenu au mot "mondialisation ». Et pour paraphraser Samuel Bordreuil, il apparaît que Bourj-Hammoud, que l'on pourrait qualifier de " quartier monde ", inscrit ceux qui y habitent, y travaillent ou le fréquentent « dans des aires d'interactions disparates, mais dont le disparate même peut pulser des connexions » pour reprendre le vocabulaire de Samuel Bordreuil, et à terme permettre de nouveaux liens. Cet espace citadin est fait de trafics, de circulations et d'affairisme et les nouvelles populations continueront probablement à l'investir dès lors qu'elles y trouveront des opportunités de gagner leur vie. Bourj-Hammoud est de ce point de vue exemplaire, il attire, comme d'autres quartiers de Beyrouth, une nouvelle maind'œuvre dont les carrières migratoires se construisent à la faveur des opportunités économiques qu'offre la ville. Elle peut venir modifier le paysage urbain par une présence plus longue et s'inscrire plus durablement dans un pays qui à terme devra compter de plus en plus avec elle.

\footnotetext{
${ }^{24}$ Samuel Bordreuil (2003) A l'orée du monde, le monde : pistes pour une phénoménologie des mondialisations, Synesthesie - Mobilités, nº 14 . http://www.synesthesie.com/mobilites/popup/bordreuil texte.html
} 